\title{
Visualization of the melanosome transfer-inhibition in a mouse epidermal cell co-culture model
}

\author{
HAE JONG KIM ${ }^{1 *}$, JULHASH U. KAZI ${ }^{2 *}$, YOU-REE LEE ${ }^{1}$, DUNG H. NGUYEN ${ }^{1}$, \\ HYANG-BOK LEE ${ }^{1}$, JEONG-HYUN SHIN ${ }^{3}$, JAE-WON SOH ${ }^{2}$ and EUN-KI KIM ${ }^{1}$ \\ ${ }^{1}$ Department of Biological Engineering, National Research Laboratory of Skin-bioactive Material; \\ ${ }^{2}$ Department of Chemistry, Biomedical Research Center for Signal Transduction Networks; \\ ${ }^{3}$ Department of Dermatology, College of Medicine, Inha University, Incheon 402-751, Korea
}

Received September 23, 2009; November 16, 2009

DOI: 10.3892/ijmm_00000337

\begin{abstract}
Transfer of melanin-containing melanosomes from melanocytes to neighboring keratinocytes results in skin pigmentation. To provide a more practical method of visualizing melanosomes in melanocytes as well as in keratinocytes, we attempted to use murine cell lines instead of human primary cells. We generated various fluorescent fusion proteins of tyrosinase, a melanin synthesis enzyme located in the melanosome, by using green fluorescent protein and red fluorescent protein. The intracellular localization of tyrosinase was then examined by fluorescence and confocal microscopy. Co-culture of murine melanocytes and keratinocytes was optimized and melanosome transfer was either stimulated with $\alpha \mathrm{MSH}$ or partially inhibited by niacinamide. To the best of our knowledge, this is the first study showing that a murine co-culture model, in addition to human primary cell co-culture, can be a good tool for depigmenting agent screening by monitoring melanosome transfer.
\end{abstract}

\section{Introduction}

Hyperpigmentation disorders, such as melasma, postinflammatory hyperpigmentation and lentigo senilis (LS), are associated with abnormal accumulation of melanin pigments, which can be improved by skin lightening reagents (1). Furthermore, Asian women prefer lighter skin color and there

Correspondence to: Dr Eun-Ki Kim, Department of Biological Engineering, Inha University, Incheon 402-751, Korea

E-mail: ekkim@inha.ac.kr

*Contributed equally

Abbreviations: GFP, green fluorescent protein; RFP, red fluorescent protein; CFDA, carboxyfluorescein diacetate; $\alpha \mathrm{MSH}, \alpha$-melanocytestimulating hormone; HTS, high throughput screening

Key words: melanin, transfer, pigmentation, melanocyte, keratinocyte, green fluorescent protein, niacinamide is a great demand to develop safer and more effective skin whitening agents (2). Although much effort has been made to develop novel therapeutic agents against pigmentation abnomalities, safer, more effective and less irritating lightening agents are still required.

To date, research on the regulation of melanogenesis has focused on factors that can inhibit tyrosinase activity and decrease total melanin content (3). Considerable knowledge of basic pigment distribution and regulation processes has been accumulated to date and should be applicable to the discovery of new targets for developing depigmenting agents.

As the present screening methods for depigmenting agents are time-consuming, rapid high-throughput screening (HTS) is required for depigmenting agent screening. Furthermore, HTS of diversified chemical libraries provide a major means for identification of lead candidates for drug or cosmetic ingredient development (4).

Visible pigmentation in mammals requires the transfer of melanin granules from melanocytes to keratinocytes. For this intercellular transfer to be effective, melanosomes must first accumulate at the distal end of the melanocyte dendrites using cooperative transport mechanisms (5). These include long range bidirectional, microtubule-dependent melanosome movements along the dendrite length before coupling to myosin Va-dependent capture machinery within the distal actin-rich regions of the dendrite (6). Myosin $\mathrm{Va}$ is recruited onto the melanosome surface by a receptor complex containing Rab27a and melanophilin in a Guanosine triphosphate (GTP)-dependent fashion (7-9). The absence of any one of these three proteins collapses the myosin Va-dependent capture of melanosomes in the periphery, causing instead their accumulation in the perikaryon of the cell.

Although melanosome transfer is an important step in pigmentation, there are few studies regarding agents used to manipulate melanosome transfer, which address the absence of an adequate in vitro screening system to measure melanosome transfer. Particularly, there is no murine in vitro screening system to measure melanosome transfer, whereas there have been many attempts to study melanosome transfer in human melanocytes and keratinocytes (10).

Therefore, this study aimed to establish a murine coculture model to screen depigmenting agents which inhibit 
melanosome transfer. In this study, we developed a murine co-culture system for screening inhibitors of melanosome transfer and confirmed its potential as an inhibitor screening tool using niacinamide, a known inhibitor of melanosome transfer.

\section{Materials and methods}

Materials. Niacinamide and $\alpha$-melanocyte-stimulating hormone $(\alpha \mathrm{MSH})$ were purchased from Sigma (St. Louis, MO, USA). Lipofectin was obtained from Invitrogen (Carlsbad, CA, USA) and CFDA from Molecular Probes. Anti-cytokeratin antibody was purchased from Zymed, anti-mouse IgG second antibody conjugated to phycoerythrin (PE) from Molecular Probes. Other chemicals were from Sigma Chemical Co. (St. Louis, MO, USA).

Cell culture. Murine cells used included pigmented Melan-A melanocytes (11) and SP-1 keratinocytes. Melan-A cells were maintained in RPMI-1640 medium supplemented with $5 \%$ fetal bovine serum (FBS), $50 \mathrm{U} / \mathrm{ml}$ penicillin, $50 \mu \mathrm{g} / \mathrm{ml}$ streptomycin, L-glutamine $0.08 \% \mathrm{w} / \mathrm{v}$ sodium bicarbonate, $200 \mathrm{nM}$ phorbol-12-myristate-13 acetate and $100 \mathrm{mM} 2$ mercaptoethanol. SP-1 keratinocytes were grown in SMEM containing $0.05 \mathrm{mM} \mathrm{Ca}^{2+}, 8 \%$ Chelex treated heat inactivated FBS, $5 \mathrm{U} / \mathrm{ml}$ penicillin and $5 \mu \mathrm{g} / \mathrm{ml}$ streptomycin. Co-culture experiments were set up according to Lei et al with an initial seeding ratio of keratinocytes and melanocytes of 5:1 in SP-1 medium (12).

GFP constructs. pGFPC3, pGFPN3 and pRFPC3 vectors were used to generate tyrosinase GFP or RFP fusion proteins which were constructed by modifying pEGFP-N1, pEGFP-C3 and pDsRed1-N1 (Clontech) vectors respectively. Vectors were generated by ligating the annealed synthetic oligonucleotides (Table I) into appropriated vector after digestion with HindIII and BamH1 and confirmed by sequencing. Human tyrosinase cDNA was the generous gift from Dr Ruth Halaban (Yale University School of Medicine). Full length open reading frame of human tyrosinase containing EcoRI cloning sites was synthesized by PCR. pGFPC3-Tyr-WT, pGFPN3-Tyr-WT and pRFPC3-Tyr-WT expression plasmids were constructed by sub-cloning the EcoRI fragment of the full length open reading frame of the human tyrosinase into pGFPC3, pGFPN3 and pRFPC 3 expression vectors respectively. All expression plasmids were verified by sequencing.

Transient transfection of melanocytes. Melan-A cells were seeded in 6-well dishes at a density of 500,000 cells/well $24 \mathrm{~h}$ prior to transfection and were transfected with Lipofectin according to the manufacturer's instructions (Invitrogen). To stimulate maturation of GFP-TYR constructs, $\alpha$ MSH (500 nM) was added $5 \mathrm{~h}$ after transfection.

Immunofluorescence staining. Melan-A cells were stained as described previously (13). Briefly, cells were transfected with TYR-GFP and fixed with $4 \%$ paraformaldehyde for $15 \mathrm{~min}$ at $4^{\circ} \mathrm{C}$. After blocking for $1 \mathrm{~h}$ at room temperature (RT), the cells were incubated with anti-cytokeratin antibody (mouse monoclonal) for $2 \mathrm{~h}$ at RT. The cells were then labeled with
PE anti-mouse (Zymed) for $1 \mathrm{~h}$ at RT. Next, cells were counterstained with DAPI and mounted. Fluorescence signals were classified into three categories, according to whether they showed green, red, or yellow fluorescence. The latter was indicative of co-localization of the red and green fluorescence signals. Images were obtained using the LSM 510 confocal microscope (Zeiss, Wetzlar, Germany).

CFDA staining. Melanocytes were stained with CFDA (Molecular Probes, Eugene, OR, USA) for $30 \mathrm{~min}$ at $37^{\circ} \mathrm{C}$, washed twice with PBS and seeded with keratinocytes as detailed before. Medium was changed after $24 \mathrm{~h}$ and testing compound, such as Niacinamide, was added. Co-cultures were trypsinized after three more days, washed once with PBS and stained with PE-anti cytokeratin antibody as detailed above. Additionally, cells were stained with the method described by Minwalla et al (10). Briefly, cells were pre-fixed in $4 \%$ paraformaldehyde (PFA), permeabilized by FACS permeabilizing solution (Becton-Dickinson, San Jose, CA, USA), incubated with primary monoclonal mouse anticytokeratin (Zymed Laboratories, San Francisco, CA, USA) at 1:300 in PBS containing 10\% human serum and $0.4 \%$ FBS for $45 \mathrm{~min}$ at RT, followed by incubation with secondary goat anti-mouse IgG antibody conjugated to phycoerythrin (PE) at a ratio of 1:20 dilution for $30 \mathrm{~min}$ at $37^{\circ} \mathrm{C}$ and post-fixation with $1 \%$ PFA.

\section{Results}

Generation of fluorescent protein fusion tyrosinase constructs. Fluorescent proteins are extensively used to visualize cellular localization and translocation of proteins. To visualize tyrosinase, we generated green fluorescent protein (GFP) and red fluorescent protein (RFP) fusion tyrosinase constructs in melanocytes or transferred melanosomes in keratynocytes. Tyrosinase is a key enzyme of melanin synthesis in melanosomes. The expression plasmids pGFPC3-hTYR-WT, pGFPN3-hTYR-WT and pRFPC3-hTYR-WT were generated by subcloning human tyrosinase cDNA into pGFPC3, pGFPN3 and pRFPC3 constructs, respectively. pGFPC3-hTYR-WT and pRFPC3-hTYR-WT plasmids encoded C-terminal GFP and RFP fusion tyrosinase proteins, respectively, while pGFPN3-hTYR-WT plasmid encoded N-terminal GFP fusion tyrosinase protein (Fig. 1A). Melan-A cells were transfected with the expression plasmids and expression of GFP or RFP fusion tyrosinase was observed by fluorescence microscopy. Cells overexpressing tyrosinase proteins did not show any morphological change (Fig. 1B). However, pGFPC3-hTYRWT, pGFPN3-hTYR-WT and pRFPC3-hTYR-WT plasmids were expressed equally and pGFPC3-hTYR-WT exhibited brighter fluorescence in Melan-A cells (Fig. 1C).

Fluorescent labeling of melanosomes. Yoon and Hearing have reported establishing a model culture system which uses immortalized murine cell lines for studies of pigmentation (14). To establish a melanosome transfer assay in murine co-culture model, we initially labeled melanocytes, Melan-A cells with a succinimidyl ester of carboxy-fluorescein diacetate (CFDA) and co-cultured with keratinocytes SP-1. These co-cultures were assessed by fluorescence microscopy for CFDA-labeled 
Table I. List of synthetic oligonucleotides.

\begin{tabular}{llcc}
\hline Vector & Parent vector & Upper strand & Lower strand \\
\hline pGFPC3 & pEGFP-N1 & 5'-AGCTTGCCACCAT & 5'-GATCGGATCCGA \\
& & GGAATTCGGATCC-3' & ATTCCATGGTGGCA-3' \\
pGFPN3 & pEGFP-C3 & 5'-AGCTTGAATTCG & 5'-GATCGATATCAG \\
& & GATCCTGATATC-3' & GATCCGAATTCA-3' \\
pRFPC3 & pDsRed1-N1 & 5'-AGCTTGCCACCAT & 5'-GATCGGATCCGAA \\
& & GGAATTCGGATCC-3' & TTCCATGGTGGCA-3' \\
\hline
\end{tabular}
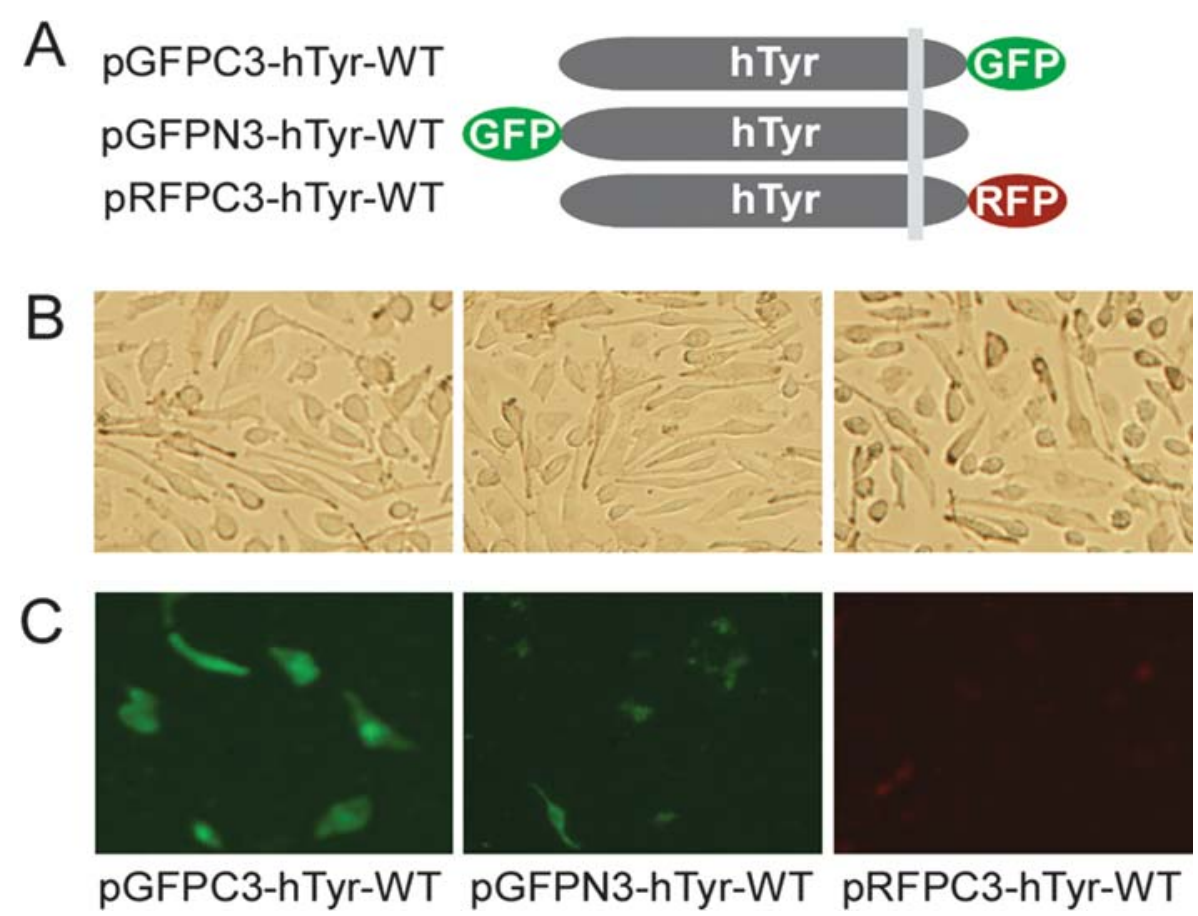

Figure 1. Construction and expression of tyrosinase plasmids. (A) Schematic representation of pGFPC3-hTYR-WT, pGFPN3-hTYR-WT and pRFPC3hTYR-WT plasmid encoded proteins. (B) Morphology of tyrosinase constructs transfected cells. (C) After transient transfection of the tyrosinase constructs, cells were grown for $48 \mathrm{~h}$ and then placed under fluorescence microscopy.

melanosome transfer in the absence (Fig. 2A) or presence (Fig. 2B) of niacinamide after 5 days. The niacinamide-treated co-culture system exhibited less CFDA-positive keratinocytes which suggests that niacinamide partially inhibited melanosome transfer from melanocytes to keratinocytes.

Tracking melanosome transfer using tyrosinase-GFP construct. To visualize melanosome transfer in Melan-A and the SP1 co-culture system, we used C-terminal GFP fusion tyrosinase constructs as a melanosome marker and cytokeratinspecific antibody as a keratynocyte marker. After transient transfection of pGFPC3-hTYR-WT into Melan-A cells, SP-1 cells were added. Cells were grown in the presence of $500 \mathrm{nM}$ $\alpha \mathrm{MSH}$ or $10 \mu \mathrm{M}$ niacinamide. Cells were fixed and SP-1 cells were labeled by anti-cytokeratin antibody followed by PEconjugated secondary antibody incubation. Cells were then placed under confocal microscopy. In $\alpha \mathrm{MSH}$-stimulated co-culture system, keratynocytes were highly populated with melanosomes (Fig. 3A), while niacinamide-treated cells contained less melanosomes (Fig. 3C). For quantification, we counted GFP-labeled melanosomes in PE-positive kerationcytes. As shown in Fig. 3D, $\alpha \mathrm{MSH}$ stimulated melanosome transfer, while niacinamide itself inhibited melanosome transfer to the keratinocytes, which is consistent with previous studies (15). This result suggests that our assay system can be applied to screen the inhibitors of melanosome transfer for depigmenting agents.

\section{Discussion}

There is a need to develop screening tools for depigmenting agents. To establish a co-culture system of murine melanocytes and keratinocytes, we labeled Melan-A cells with GFP and conducted co-culturing with keratinocytes.

Our in vitro model system involving co-cultures allowed us to investigate the interactions between melanocytes and keratinocytes. Niacinamide added to the system was shown to effectively inhibit melanosome transfer by confocal 

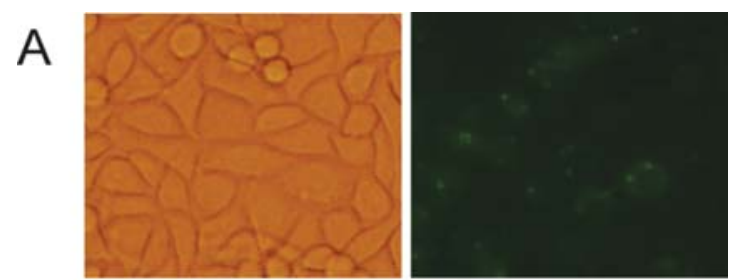

B
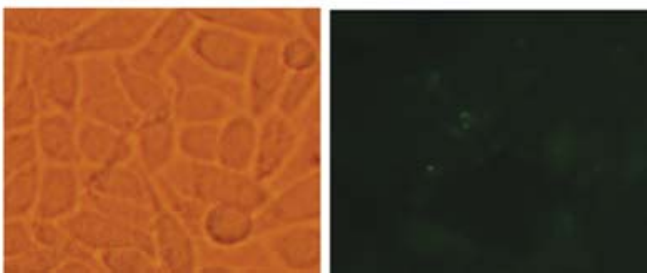

Figure 2. CFDA-labeled Melan-A and SP-1 cells co-culture. Melan-A cells were labeled by CFDA and mixed with SP-1 cells. Cells were grown for five days in absence (A) or presence (B) of niacinamide and then placed under fluorescence microscopy.

microscopy. Our investigations using confocal microscopy demonstrated that melanosomes are transferred from melanocytes to keratinocytes in our model system. We successfully labeled Melan-A cells with a fluorochrome, CFDA, prior to co-culturing them with SP-1 cells. Our investigations using scanning laser confocal microscopy demonstrate that the fluorescent-labeled melanocytes transferred fluorochrome to surrounding keratinocytes within a 5-day period. We quantified this transfer by counting the
GFP-positive spots in adjacent keratinocytes and then subsequently quantified the effectiveness of exogenously added niacinamide which inhibited the transfer of the fluorochrome to keratinocytes.

Recently, Singh et al have developed a novel in vitro assay that exploits the specificity of Silv/Pmel17/gp100 expression for melanosome/melanin granules (15). To measure the melanosome transfer quantitatively, they used double immunolabelling with anti-gp100 antibody (green) and anticytokeratin antibody (red) instead of GFP transfection (green) and anti-cytokeratin antibody followed PE staining (red) employed in our system. Using CFDA and the PE-system another group showed that $10 \mu \mathrm{M}$ niacinamide was able to inhibit 21 to $32 \%$ of melanosome transfer (16) while we observed similar inhibition using GFP and PE system. These results suggest that our system is suitable for the quantification of melanosome transfer.

To evaluate this murine co-culture model as an HTS tool for depigmenting agents, we determined whether a known melanosome transfer inhibitor, niacinamide, inhibited melanosome transfer in our system. In conclusion, we report that murine co-culture model is an HTS tool for screening depigmenting agents.

\section{Acknowledgements}

This study was supported by the Korea Science and Engineering Foundation (KOSEF) grant funded by the Korea Government (MEST) R0A-2007-000-10015-0.
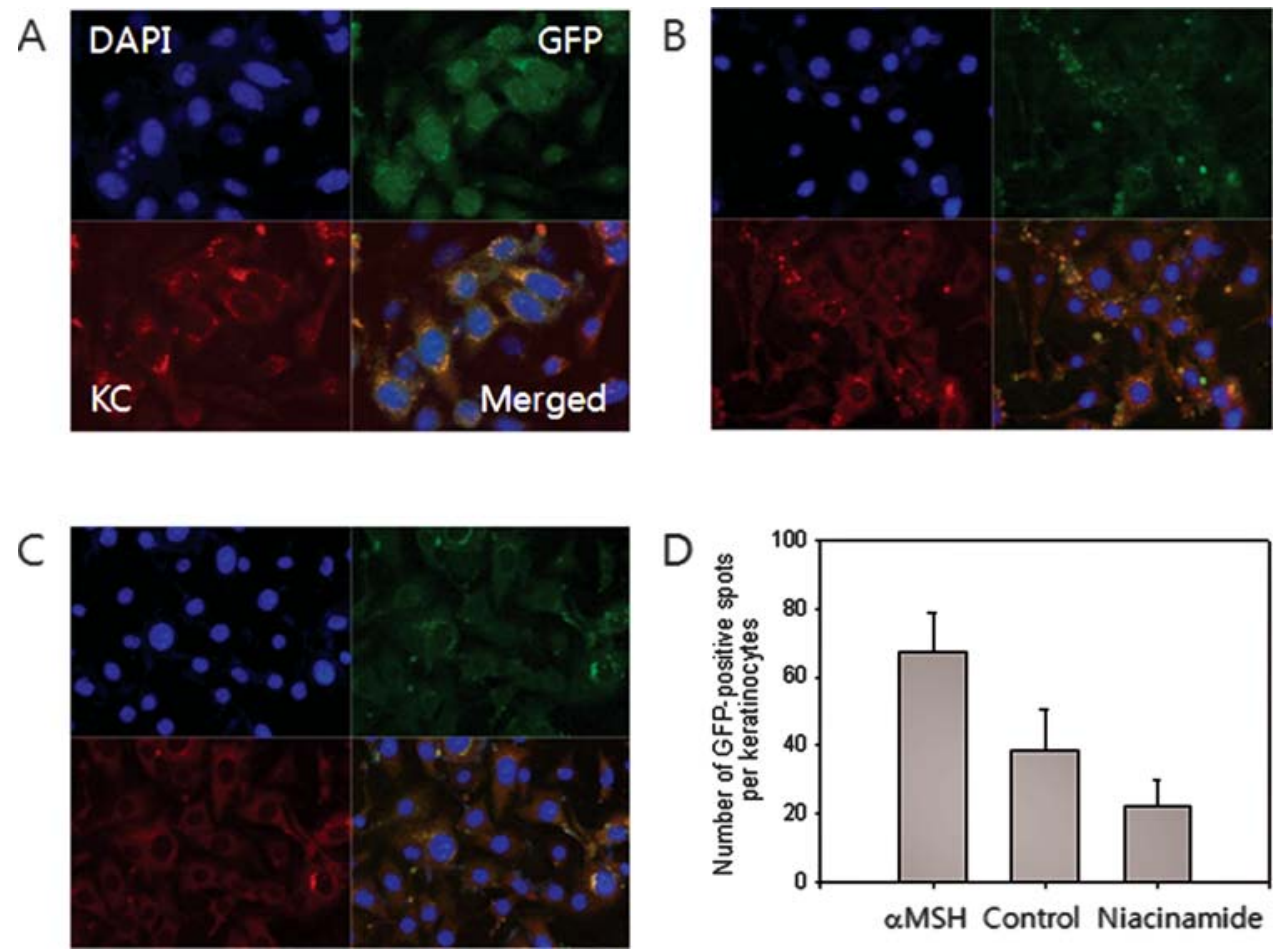

Figure 3. Visualization of melanosome inhibition. pGFPN3-hTYR-WT-transfected Melan-A cells were mixed with SP-1 cells. After five days of co-culture treated with $500 \mathrm{nM} \alpha \mathrm{MSH}$ (A), vehicle (B), or $10 \mu \mathrm{M}$ niacinamide (C), cells were fixed and incubated with anti-cytokeratin antibody followed by phycoerythrine (PE)-conjugated anti-mouse antibody incubation. Cells were then observed by confocal microscopy. Merged image revealed quantifiable intra-cytoplasmic and peri-nuclear GFP-positive spots in adjacent keratinocytes. (D) Quantification of transferred melanosomes taken from five randomly selected microscopic fields (total 20 cells per field) for each of the different treatment groups. KC refers to keratinocytes visualized by anti-cytokeratin antibody and PE-conjugated secondary antibody. 


\section{References}

1. Ito Y, Kanamaru A and Tada A: Effects of methylophiopogonanone B on melanosome transfer and dendrite retraction. J Dermatol Sci 42: 68-70, 2006.

2. Zhong S, Wu Y, Soo-Mi A, et al: Depigmentation of melanocytes by the treatment of extracts from traditional Chinese herbs: a cell culture assay. Biol Pharm Bull 29: 1947-1951, 2006.

3. Hwang JS, Choi H, Rho HS, et al: Pigment-lightening effect of $\mathrm{N}, \mathrm{N}^{\prime}$-dilinoleylcystamine on human melanoma cells. Br J Dermatol 150: 39-46, 2004.

4. Horiuchi KY, Wang Y, Diamond SL, et al: Microarrays for the functional analysis of the chemical-kinase interactome. J Biomol Screen 11: 48-56, 2006.

5. Seiberg M: Keratinocyte-melanocyte interactions during melanosome transfer. Pigment Cell Res 14: 236-242, 2001.

6. Wu X, Bowers B, Rao K, et al: Visualization of melanosome dynamics within wild-type and dilute melanocytes suggests a paradigm for myosin V function in vivo. J Cell Biol 143: 1899-1918, 1998.

7. Fukuda M, Kuroda TS and Mikoshiba K: Slac2-a/melanophilin, the missing link between Rab27 and myosin Va: implications of a tripartite protein complex for melanosome transport. J Biol Chem 277: 12432-12436, 2002.

8. Strom M, Hume AN, Tarafder AK, et al: A family of Rab27binding proteins. Melanophilin links Rab27a and myosin Va function in melanosome transport. J Biol Chem 277: 25423-25430, 2002.
9. Wu XS, Rao K, Zhang H, et al: Identification of an organelle receptor for myosin-Va. Nat Cell Biol 4: 271-278, 2002.

10. Minwalla L, Zhao Y, Cornelius J, et al: Inhibition of melanosome transfer from melanocytes to keratinocytes by lectins and neoglycoproteins in an in vitro model system. Pigment Cell Res 14: 185-194, 2001.

11. Bennett DC, Cooper PJ and Hart IR: A line of non-tumorigenic mouse melanocytes, syngeneic with the B16 melanoma and requiring a tumour promoter for growth. Int J Cancer 39: 414-418, 1987.

12. Lei TC, Virador VM, Vieira WD, et al: A melanocyte-keratinocyte coculture model to assess regulators of pigmentation in vitro. Anal Biochem 305: 260-268, 2002.

13. Toyofuku K, Wada I, Valencia JC, et al: Oculocutaneous albinism types 1 and 3 are ER retention diseases: mutation of tyrosinase or Tyrp1 can affect the processing of both mutant and wild-type proteins. FASEB J 15: 2149-2161, 2001.

14. Yoon TJ and Hearing VJ: Co-culture of mouse epidermal cells for studies of pigmentation. Pigment Cell Res 16: 159-163, 2003.

15. Singh SK, Nizard C, Kurfurst R, et al: The silver locus product (Silv/gp100/Pmel17) as a new tool for the analysis of melanosome transfer in human melanocyte-keratinocyte co-culture. Exp Dermatol 17: 418-426, 2008.

16. Greatens A, Hakozaki T, Koshoffer A, et al: Effective inhibition of melanosome transfer to keratinocytes by lectins and niacinamide is reversible. Exp Dermatol 14: 498-508, 2005. 